\title{
MECHANIZATION OF SCORE PROCESSING IN A FRESHMAN TESTING PROGRAM ${ }^{1}$
}

\author{
JASON MILLMAN \\ University of Michigan \\ AND \\ RUSSELL MILLMAN \\ Cincinnati, Ohio

\section{Introduction}

LIKE many universities and colleges, each fall the University of Michigan administers a battery of achievement and aptitude tests to its incoming freshmen. These tests are scored on a conventional IBM scoring machine. The purpose of this paper is to describe a new procedure, followed successfully this past year, in the processing of these test scores.

We believe this procedure is applicable to schools which score tests of a larger number of students (say 1,000 or more), using an IBM scoring machine, and wish to do some or all of the following:

1. Operate on the machine scores to produce other sources, such as "total" or "accuracy" scores.

2. Convert scores to desired norm values.

3. Classify students into special programs or courses (e.g., remedial courses) based on predetermined combinations of their test scores.

4. Prepare for each student a profile sheet to show his scores.

5. Prepare for each student an IBM card containing his scores.

6. Calculate test statistics broken down by subgroups if desired.

7. Predict grades using a predetermined weighting of the tests.

In connection with its freshmen testing program, the University does all these operations with the exception of predicting grades

\footnotetext{
1 We wish to thank Dr. John Milholland, Chief of the University of Michigan's Evaluation and Examinations Division, for his encouragement of this undertaking and for his helpful review of the manuscript.
} 
(number 7). In the past they have been done entirely by hand, with the calculation of test statistics (number 6 ) being completed about one-half year after the tests have been administered and the preparation of the IBM card (number 5) within a month.

Numbers 1, 2, 3, and 4 are completed 15 hours after the last test has been administered. To accomplish this in former years a large staff of temporary student help, not often interested nor responsible in these matters, worked around the clock. This strictly clerical method proved inefficient, inaccurate, and uneconomical compared to the method to be described.

The new procedure is able to do all the operations within 15 hours after the last test has been administered. It makes use of the IBM 650 Magnetic Drum Computer. Since the main program can process a student in a couple of seconds, it would probably be worthwhile to rent time on such a machine from a data processing company if one is not available at the college or university.

\section{Outline of the Procedure}

The tests are scored, as before, using IBM test scoring machines. Usually 6 to 8 machine scores on 4 or 5 answer sheets are obtained for each student. These scores, together with a student identification number obtained from a gummed label pasted on each answer sheet and a test identification number, are punched into IBM cards, one card for each answer sheet. (Scores from almost 1,000 answer sheets can be punched in an hour.) These are called test cards.

The only completely hand operation consists of posting the gummed label number on an interpreted IBM card containing the student's name, other identifying information, and high school percentile rank. These cards, called master cards, are obtained from the Office of Admissions. The gummed label number is subsequently punched into the master card. (Where cards are not available the student's name and other desired non-test information might be taken from a questionnaire to be filled out by the student in the testing session. For efficiency the questionnaire might be printed on an IBM card.)

With the gummed label number as the control, master and test cards are merged mechanically by the Collator. The merged decks serve as the input for the 650 . The 650 then computes the "total" and "accuracy" scores where applicable, converts all raw scores to 
percentile rank equivalents, and determines which students meet the test score criteria for the various special courses. The output for each student consists of a freshman card together with one or more classification cards where appropriate.

The freshman cards are first used as the input of an auxiliary program which performs the statistical analysis. The particular analysis employed provides summary test statistics (number of cases, means, and standard deviations) for each unit within the University and for all units combined. In addition statistics are calculated for male, female, instate, and outstate students within each unit and for all units combined.

The freshman cards are next reproduced into preprinted blank cards, interpreted and are ready for distribution to the various campus agencies which use them. Although not done this year, a set of these cards could be put through a tabulator and, using continuous form profile sheets, the test scores and student information could be printed on these profile sheets.

The classification cards are sorted, alphabetized, and simple listings made after the appropriate header cards.

One thousand students, with an average of four and one-half answer sheets each, can be processed through the main program of the 650 in forty minutes and through the auxiliary program in twenty-five minutes. Complete test data in every card are not required for either the main or auxiliary program. A student may be missing none, some, or all test scores. 